\title{
STUDY OF Hg VACANCIES IN (Hg,Cd)Te AFTER THM GROWTH AND POST-GROWTH ANNEALING BY POSITRON ANNIHILATION
}

\section{R. KRAUSE}

Sektion Physik, Martin-Luther-Universität Halle, DDR-4010 Halle, German Dem. Rep.

\section{A. KLIMAKOW and F.M. KIESSLING}

Sektion Physik, Humboldt-Universität Berlin, Invalidenstrasse 110, DDR-1040 Berlin, German Dem. Rep.

\author{
A. POLITY \\ Sektion Physik, Martin-Luther-Universität Halle, DDR-4010 Halle, German Dem. Rep.
}

and

\section{P. GILLE and M. SCHENK}

Sektion Physik, Humboldt-Universität Berlin, Invalidenstrasse 110, DDR-1040 Berlin, German Dem. Rep.

Positron lifetime measurements have been performed to study vacancy defects in $\mathrm{Hg}_{0.78} \mathrm{Cd}_{0.22} \mathrm{Te}$. Post-growth annealing under various $\mathrm{Hg}$ vapour pressure conditions have been used to create a well-defined number of $\mathrm{Hg}$ vacancies. The sensitivity range of the positron annihilation method was found to be $10^{15}<c_{\mathrm{vac}}^{\mathrm{Hg}}<10^{18} \mathrm{~cm}^{-3}$. The obtained experience has been used to investigate THM-grown single crystals. The measured longitudinal and radial dependence of the vacancy concentration can be explained by the temperature profile in the grown $(\mathrm{Hg}, \mathrm{Cd}) \mathrm{Te}$ ingots.

\section{Introduction}

Because of their high mobility, $\mathrm{Hg}$ vacancies are the most important native point defects in $\mathrm{Hg}_{1-x} \mathrm{Cd}_{x} \mathrm{Te}$ (MCT). They act as acceptors and influence or even dominate the electrical behaviour of the material. Thus $\mathrm{Hg}$ vacancies may in principle be studied by Hall effect measurements, taking into account that the measured number of carriers is the sum effect of all electrically active native donors and acceptors as well as doping impurities present in the sample. Therefore, measurements of the $\mathrm{Hg}$ vacancy concentration with an independent method combined with Hall measurements should improve the knowledge about the point defect behaviour in MCT.

Positron annihilation is a well-established method to identify vacancy defects in solids [1]. While a number of studies has been done in the field of $A_{3} B_{5}$ compounds (see the review [2]), no systematic investigations have been performed for studying the vacancies in MCT by positrons. In the present paper, therefore, we demonstrate first of all the sensitivity of the method to detect $\mathrm{Hg}$ vacancies in MCT. For this purpose, post-growth annealings were carried out under various $\mathrm{Hg}$ vapour conditions to create a well defined number of $\mathrm{Hg}$ vacancies. Secondly, we show applications of this method to determine the number and distribution of $\mathrm{Hg}$ vacancies after THM growth and attribute them to the growth conditions.

\section{Positron annihilation technique}

The most powerful positron technique for studying bulk defects is the lifetime spectroscopy. The positrons are obtained by a weak $(15 \mu \mathrm{Ci})$ 
${ }^{22} \mathrm{NaCl}$ source within two thin $(3 \mu \mathrm{m}) \mathrm{Al}$ foils of $4 \times 4 \mathrm{~mm}^{2}$ in size (active area $2 \times 2 \mathrm{~mm}^{2}$ ). The source is placed between an identical pair of samples (two pieces of a wafer or two neighbouring wafers). The lifetime of a single event is measured by detecting the time difference between the birth gamma $(1.28 \mathrm{MeV}$, appearing in the source together with the positron) and the annihilation gamma $(0.51 \mathrm{MeV})$. Within $2 \mathrm{~h}$, about 2 million counts are collected to form the lifetime spectrum, which is analyzed in one or two components (sum of exponentials).

When the positrons penetrate into the sample, their high kinetic energy (up to $0.54 \mathrm{MeV}$ ) is lost within a few picoseconds. The positron starts to diffuse through the bulk of the sample and in absence of any defects it annihilates after its lifetime with an electron. The lifetime spectrum consists of one component: $\tau=\tau_{\mathrm{b}}$ (where the subscript b stands for bulk; $\tau_{b}$ is constant, $\tau_{b}=264$ ps for $\mathrm{Hg}_{0.78} \mathrm{Cd}_{0.22} \mathrm{Te}$ ). In the case of vacancies present in the sample, the positrons may be trapped there until they annihilate. Because of the decreased electron density within the defect, the positron lifetime will be increased and the lifetime spectrum consists of two exponential decay components. From the obtained fitting parameters $\tau_{1}$ and $\tau_{2}$, and $I_{2}$ (lifetime components and intensities), the positron trapping rate $\kappa$, which is proportional to the vacancy concentration $c_{v}$, may be determined

$$
\begin{aligned}
& \tau_{1}=\frac{1}{\lambda_{\mathrm{b}}+\kappa}, \quad \tau_{2}=\frac{1}{\lambda_{\mathrm{d}}}, \\
& I_{2}=\frac{\kappa}{\lambda_{\mathrm{b}}+\kappa-\lambda_{\mathrm{d}}}, \quad I_{1}=1-I_{2},
\end{aligned}
$$

where the subscript $\mathbf{d}$ stands for defect. The annihilation rates $\lambda_{i}$ are defined as the reciprocal lifetimes $\lambda_{\mathrm{i}}=1 / \tau_{\mathrm{i}}$.

One may also use the average lifetime $\bar{\tau}$ to determine the trapping rate $\kappa$, because $\bar{\tau}$ is rather insensitive to numerical uncertainties of the decomposition procedure:

$\bar{\tau}=I_{1} \tau_{1}+I_{2} \tau_{2}, \quad \kappa=\lambda_{\mathrm{b}} \frac{\bar{\tau}-\tau_{\mathrm{b}}}{\tau_{\mathrm{d}}-\bar{\tau}}$.

The constant lifetimes $\tau_{\mathrm{b}}$ and $\tau_{\mathrm{d}}$ may in principle be obtained by a single measurement, but they are usually extracted after a set of experiments $\left(\tau_{\mathrm{d}}\right.$ is a function of the type of defect, but not of its concentration; $\tau_{\mathrm{d}}=298 \mathrm{ps}$ for $\mathrm{Hg}$ monovacancies in $\mathrm{Hg}_{0.78} \mathrm{Cd}_{0.22} \mathrm{Te}$ ). Thus the positron trapping rate $\kappa$ delivers the vacancy concentration $c_{\mathrm{v}}$ if the proportionality constant $\mu_{\mathrm{v}}$ could be obtained at least once by an independent method:

$\kappa=\mu_{\mathrm{v}} c_{\mathrm{v}}$

The so-called specific positron trapping rate $\mu_{v}$ is a constant for a given type of defect at certain temperature.

In a semiconductor, the vacancies may carry a positive or negative charge that will lead to an additional coulombic tail of the potential responsible for the positron trapping. Thus no positron trapping is expected for positively charged vacancies, because they repel positrons. On the other hand, the specific positron trapping rate is expected to be larger for negatively charged than for neutral defects. The $\mathrm{Hg}$ vacancies in MCT are negatively charged in the whole temperature range of interest (see the discussion in section 3). The determination of the specific positron trapping rate for a compound semiconductor is difficult because of the lack of an independent method to measure the vacancy concentration (see the discussion in ref. [2]). We will show later that the post-growth annealing of MCT gives, for the first time, the unique possibility to determine this constant in a compound semiconductor with high precision.

\section{Hg vacancies after post-growth annealing}

The single crystals of $\mathrm{Hg}_{0.78} \mathrm{Cd}_{0.22} \mathrm{Te}$ used for the post-growth annealing under various $\mathrm{Hg}$ vapour pressure conditions have been obtained by THM growth (travelling heater method) from the Te-rich solution. The principle of this method for growing MCT crystals has been described in [3]. The annealing has been performed using the closed-tube method. The sample thickness to be treated was $500 \mu \mathrm{m}$. The $\mathrm{Hg}$ vapour pressure during an anneal event was controlled by a $\mathrm{Hg}$ 


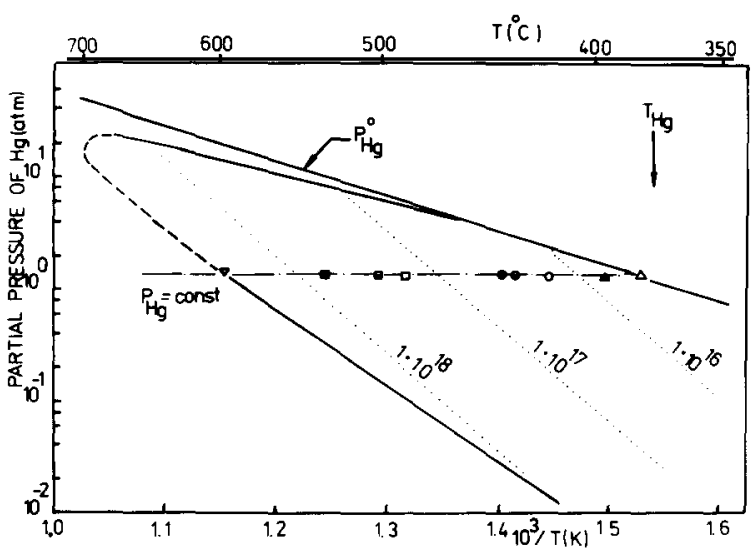

Fig. 1. Mercury pressure over solid $\mathrm{Hg}_{0.78} \mathrm{Cd}_{0.22} \mathrm{Te}$ as a function of sample temperature [4]. Dotted lines are iso-concentration lines (calculated hole concentrations $p_{77}$ ). The sample temperature is given on the abscissa.

reservoir held at $375^{\circ} \mathrm{C}$ corresponding to a pressure of $1.4 \mathrm{~atm}$. The sample was kept at the other end of the quartz tube at distinct temperature controlling the required vacancy concentration (two-temperature annealing). The points marked in fig. 1 indicate different annealing experiments. If one follows simple thermodynamical equations [4] taking into account only $\mathrm{Hg}$ vacancies as electrically active point defects, the number of charged carriers at deep temperatures can be derived. The dotted lines in fig. 1 and the dashed line in fig. 2 correspond to the values calculated on the basis of the defect model as a function of the sample temperature. The satisfactory agreement with the experimental points (Hall measurements) indicates the validity of the assumption mentioned above, i.e., the $\mathrm{Hg}$ vacancies dominate the electrical behaviour. Concerning our temperature-dependent Hall measurements, a high degree of compensation of donors and acceptors is not probable. For a detailed discussion, see the more extended papers [5] and [6]. We conclude that it is possible to change the number of $\mathrm{Hg}$ vacancies in a controlled way.

This is the essential supposition to scale the sensitivity range of the positron lifetime spectroscopy. Therefore, a set of samples have been measured with positrons (see fig. 3). The n-type sample and the samples with a small number of holes $p_{77}$ (which equals in this case the vacancy

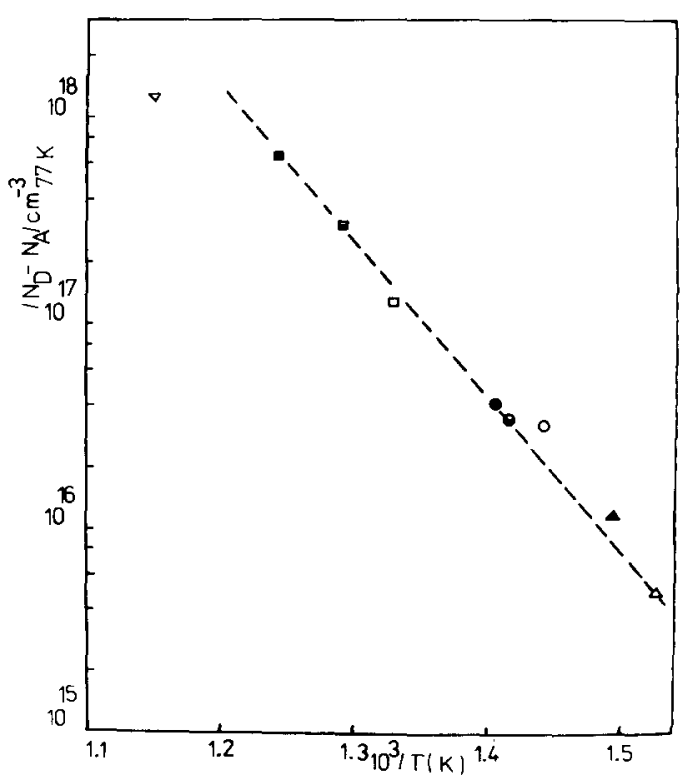

Fig. 2. Hole concentration $p_{77}$ as a function of sample temperature $\left(\boldsymbol{P}_{\mathrm{Hg}}=\right.$ constant-1.4 $\left.\mathrm{atm}\right)$ during post-growth annealing. The dashed line represents the destination curve for the annealing corresponding to thermodynamical calculation taking into account only $\mathrm{Hg}$ vacancies but no other donors or acceptors.

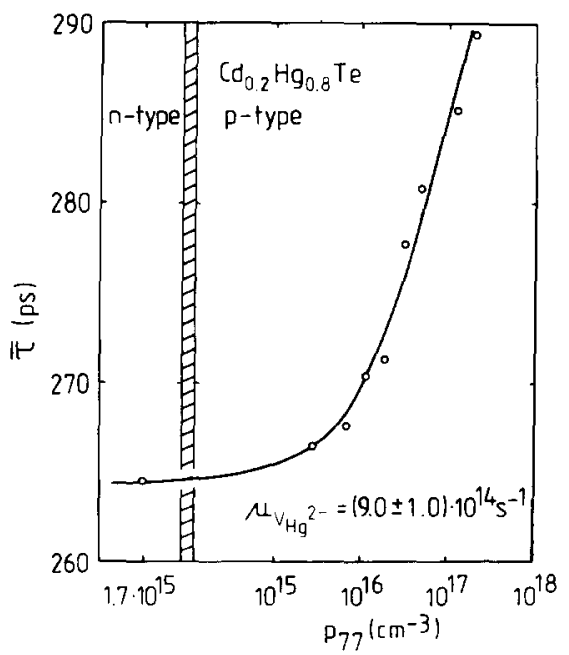

Fig. 3. The average positron lifetime $\bar{\tau}$ versus hole concentration $p_{77}$ for differently annealed samples. The number of holes equals, in these samples, the number of $\mathrm{Hg}$ vacancies. Therefore the obtained line represents the sensitivity curve of the positron annihilation. The solid line and the specific positron trapping rate $\mu_{v}^{\mathrm{Hg}}=(6.1 \pm 0.5) \times 10^{-8} \mathrm{~cm}^{3} \mathrm{~s}^{-1}$ were found by a fit to the data using eqs. (1) and (2). 
concentration $\left.c_{\mathrm{vac}}^{\mathrm{Hg}}\right)$ do not show any positron trapping $\left(\tau=\tau_{b}=264 \pm 1\right.$ ps). With increasing number of vacancies the average positron lifetime $\bar{\tau}$ also increases due to the growing number of the positrons trapped by vacancies. The spectra consist of two components with the defect lifetime $\tau_{\mathrm{d}}=298 \pm 3 \mathrm{ps}$ which is independent of the defect concentration (compare eq. (1)). Using eqs. (2) and (3), a simple fit delivers the desired specific positron trapping rate $\mu_{\mathrm{v}}^{\mathrm{Hg}}$ with an extraordinary accuracy compared to earlier estimations in compound semiconductors: $\mu_{\mathrm{v}}^{\mathrm{Hg}}=(6.1 \pm 0.5) \times 10^{-8}$ $\mathrm{cm}^{3} \mathrm{~s}^{-1}=(9.0 \pm 1.0) \times 10^{14} \mathrm{~s}^{-1}$. The Hall experiments have been done at $77 \mathrm{~K}$, while the positron lifetime measurements were performed at room temperature (RT) where the sample is in a different state, because the conduction type of the sample has been converted at RT to n-conductivity. The effect of temperature on the positron trapping has been carefully checked. From temperaturedependent lifetime experiments [5], we conclude that the specific positron trapping rate does not change between 100 and $300 \mathrm{~K}$. Therefore, the obtained value $\mu_{\mathrm{v}}^{\mathrm{Hg}}$ is valid in the whole temperature range and leads to the sensitivity range of the positron annihilation method of $10^{15}<c_{\mathrm{vac}}^{\mathrm{Hg}}<10^{18}$ $\mathrm{cm}^{-3}$. See the more detailed discussion in ref. [5].

\section{Hg vacancies after THM growth}

( $\mathrm{Hg}, \mathrm{Cd}$ )Te crystals have been grown at the $\mathrm{Hg}$ deficiency side of the phase diagram. Five complete as-grown THM single crystals $(x=0.22)$ have been investigated by positron lifetime spectroscopy and Hall measurements after having been cut into $1.2 \mathrm{~mm}$ thick slices. The lifetime has been measured in the center of all neighbouring wafers and in addition in a radial direction on some selected pairs of wafers. Fig. 4 and fig. 5 show typical results for an intentionally undoped crystal. The vacancy concentration is low (less than $5 \times$ $10^{15} \mathrm{~cm}^{-3}$ ) in the whole length of the crystal except at the end where the concentration increases to $c_{\text {vac }}^{\mathrm{Hg}}=1.5 \times 10^{17} \mathrm{~cm}^{-3}$. The hole concentration $p_{77}$ shows the same features. It is low at the beginning indicating the low content of doping impurities and $\mathrm{Hg}$ vacancies. It increases

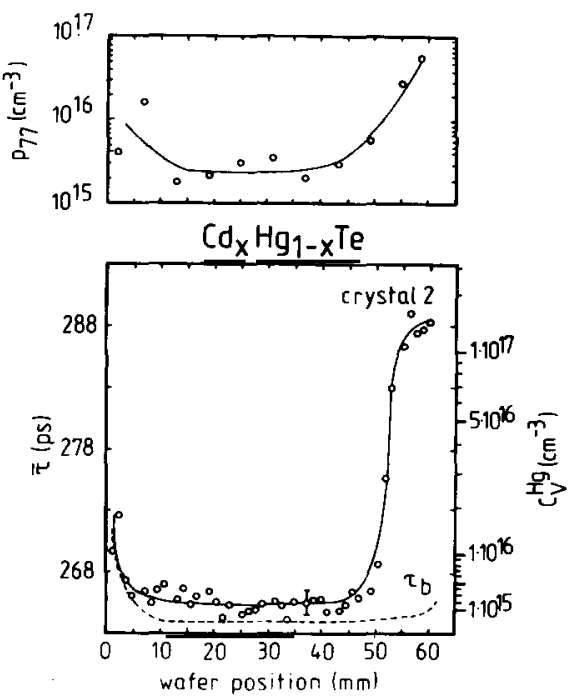

Fig. 4. The average positron lifetime $\bar{\tau}$ and the hole concentration $p_{77}$ measured in the center of neighbouring wafers at given positions in the $\mathrm{Hg}_{1-x} \mathrm{Cd}_{x} \mathrm{Te}$ ingot. At the right margin the $\mathrm{Hg}$ vacancy concentration is scaled. This axis is valid in the case that the $x$ value, and therefore the bulk lifetime (dashed

line), is constant, $\tau_{b}=264$ ps ( $5 \mathrm{~mm}$ beyond the seed).

simultaneously to the $\mathrm{Hg}$ vacancy concentration because each vacancy acts as charged acceptor at $77 \mathrm{~K}$.

The increase of the $\mathrm{Hg}$ vacancy concentration

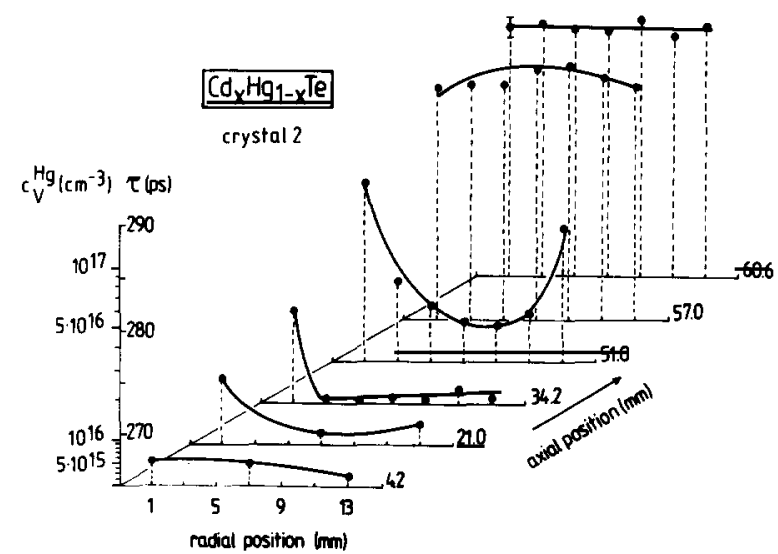

Fig. 5. The average positron lifetime $\bar{\tau}$ in radial dependence on some selected $\mathrm{Hg}_{1-x} \mathrm{Cd}_{x} \mathrm{Te}$ wafers of the same ingot. The axial separation was not equidistant (marked in the figure). At the ordinate, the $\mathrm{Hg}$ vacancy concentration is drawn in addition. This axis is valid for the upper five curves where the $x$ value remains constant, $x=0.22$. 
at the end of each crystal can be correlated to the typical longitudinal and radial temperature distribution in the crystal caused by the THM arrangement. With the very low growth rate (typically 1 $\mathrm{mm}$ day $^{-1}$ ), a temperature-depending $\mathrm{Hg}$ vacancy concentration is established corresponding to the solubility limit of the existence region $[7,8]$. These results have also been found with a very short crystal having the same thermal history as the last part of a longer one. The cooling behaviour is caused by the strong decrease in the axial temperature profile according to the used one-zone furnace. During cooling down the heater and the grown crystal after having finished the growth process a high number of $\mathrm{Hg}$ vacancies has been frozen in. Depending on the cooling regime the radial defect profile (fig. 5) is also to be understood, i.e. after cooling down the heater with a rate of $30 \mathrm{~K} \mathrm{~h}^{-1}$, the profiles have a U-shape [6].

The high vacancy concentration and all the measured profiles can be levelled by post-growth annealing under $\mathrm{Hg}$ atmosphere and the desired $\mathrm{Hg}$ vacancy concentration is adjustable. This has been shown by measuring as-grown wafers before and after the following annealing procedure [6].

\section{Conclusions}

$\mathrm{Hg}$ vacancies in $\mathrm{Hg}_{1-x} \mathrm{Cd}_{x} \mathrm{Te}$ are highly mobile and electrically active. Therefore, they are the most important native point defects of this material.

Positron annihilation spectroscopy can identify the $\mathrm{Hg}$ vacancies. The specific positron trapping rate, being a constant connecting the experimentally obtained trapping rate $\kappa$ with the required value of the $\mathrm{Hg}$ vacancy concentration, could be determined to be $\mu_{\mathrm{v}}^{\mathrm{Hg}}=(6.1 \pm 0.5) \times 10^{-8} \mathrm{~cm}^{3} \mathrm{~s}^{-1}$. Thus the sensitivity of the method range is $10^{15}<$ $c_{\text {vac }}^{\mathrm{Hg}}<10^{18} \mathrm{~cm}^{-3} \cdot \mu_{\mathrm{v}}^{\mathrm{Hg}}$ is temperature-independent in the range $100<T<300 \mathrm{~K}$ and is also independent of the conduction type.

Five complete $\mathrm{Hg}_{1-x} \mathrm{Cd}_{x} \mathrm{Te}$ single crystals have been studied in the as-grown state. The $\mathrm{Hg}$ vacancy concentration profiles can be explained by the cooling behaviour of the crystal during growth (frozen-in vacancies). Post-growth annealing under $\mathrm{Hg}$ atmosphere following the growth may completely level the $\mathrm{Hg}$ vacancy distribution.

\section{Acknowledgements}

The authors wish to thank Dr. W. Hörstel (Humboldt-Universität Berlin, Sektion Physik) for the carefully performed Hall measurements and helpful discussions.

\section{References}

[1] P. Hautojärvi, Ed., Positrons in Solids, Topics in Current Physics, Vol. 12 (Springer, Heidelberg, 1979).

[2] G. Dlubek and R. Krause, Phys. Status Solidi (a) 102 (1987) 443

[3] R. Triboulet, T. Nguyen Duy and A. Durand, J. Vacuum Sci. Technol. A3 (1985) 95.

[4] H.R. Vydganath, J. Electrochem. Soc. 128 (1981) 2609.

[5] R. Krause, A. Polity, A. Klimakow and W. Hörstel, A positron study of $\mathrm{Hg}$ vacancies in $\mathrm{Hg}_{1-x} \mathrm{Cd}_{x} \mathrm{Te}$, Part $1: \mathrm{Hg}$ vacancies after post-growth annealing, to be published.

[6] R. Krause, F.M. Kiessling, A. Polity, P. Gille and M. Neubert, A positron study of $\mathrm{Hg}$ vacancies in $\mathrm{Hg}_{1-x} \mathrm{Cd}_{x} \mathrm{Te}$, Part 2: $\mathrm{Hg}$ vacancies after THM growth, to be published.

[7] H.F. Schaake, J. Electron. Mater. 14 (1985) 513.

[8] T.C. Harman, J. Vacuum Sci. Technol. As (1987) 3055. 